\title{
Effect of Magnetic Property Modification on Current-Induced Magnetization Switching with Perpendicular Magnetic Layers and Polarization-Enhancement Layers
}

\author{
Woojin Kim ${ }^{1}$, Kyung-Jin Lee ${ }^{2}$, and Taek Dong Lee ${ }^{1 *}$ \\ ${ }^{1}$ Department of Materials Science and Engineering, KAIST, Daejeon 305-701, Korea \\ ${ }^{2}$ Department of Materials Science and Engineering, Korea University, Seoul 136-713, Korea
}

(Received 15 January 2009, Received in final form 30 March 2009, Accepted 8 April 2009)

\begin{abstract}
The effects of the magnetic property variation on current-induced magnetization switching in magnetic tunnel junction with perpendicular magnetic anistoropy (PMA) and the soft magnetic polarization-enhancement layers (PELs) inserted between the layers with PMA and the MgO layer was studied. A micromatnetic model was used to estimate the switching time of the free layer by different applied current densities, with changing saturation magnetization $\left(M_{s}\right)$ of the PELs, interlayer exchange coupling between PMA layers and PELs. The switching time could be significantly reduced at low current densities, by increasing $M_{s}$ of PELs and decreasing interlayer exchange coupling.
\end{abstract}

Keywords : current-induced magnetization switching, perpendicular magnetic anisotropy, micromagnetics, magnetic random access memory

\section{Introduction}

A magnetic tunnel junction (MTJ) with perpendicular magnetic anisotropy (PMA) was proposed as a candidate for high-density magnetic random access memory (MRAM) [1]. This resolves the problem of insufficient thermal stability due to volume reduction of the magnetic cell for high density. However, since a high magneto-resistance ratio is essential for the application, coherent tunneling of spin-polarized electrons must occur at the interface of the $\mathrm{MgO}$ layer and adjacent magnetic layer [2, 3]. Therefore, soft magnetic polarization-enhancement layers (PELs) are generally inserted between the magnetic layers with PMA and $\mathrm{MgO}$ layer, to enhance the coherent tunneling of the spin current $[1,4]$.

Spin-transfer torque provides the manipulation of magnetization by spin-polarized current, without the magnetic field $[5,6]$. Current-induced magnetization switching (CIMS) is believed to be the promising write scheme for MRAM. The switching current density of a single magnetic layer is theoretically given by the single domain model [7]. However, the single domain model fails to describe the switching of the magnetic cell if the cell

*Corresponding author: Tel: +82-42-350-3336

Fax: +82-42-350-5310, e-mail: tdlee@kaist.ac.kr consists of magnetic layers with different properties. Herein, we report the effects of magnetic property variations on the CIMS in MTJs with PMA and PEL's in terms of switching time, using the micromagnetic model.

\section{Model and Method}

The electric current of a 10-ns pulse was applied to cylindrical MTJs with a diameter of $40 \mathrm{~nm}$, consisting of the pinned layer (PL (20 nm))|Bottom PEL (1 nm)|MgO $(1 \mathrm{~nm}) \mid$ Top PEL $(1 \mathrm{~nm}) \mid$ free layer $(\mathrm{FL}(6 \mathrm{~nm}))$. In the rest of the paper, the layer of 'PL/Bottom PEL' will be referred to as the composite pinned layer (CmPL), and the layer of 'Top PEL/FL' as the composite free layer (CmFL)'. The MTJs are discretized into unit cells of $2 \mathrm{~nm} \times 2 \mathrm{~nm} \times$ $1 \mathrm{~nm}$. A MTJ of default magnetic properties was established; when one property was changed, the rest of the properties were fixed as the default values. The default MTJ has the following properties. The PL and FL with a PMA of $2.0 \times 10^{6} \mathrm{erg} / \mathrm{cm}^{3}$, and $M_{\mathrm{s}}$ of $400 \mathrm{emu} / \mathrm{cm}^{3}$. A magnetic material with such a $M_{s}$ and PMA can be obtained in various $\mathrm{Fe}$ or Co alloys [8]. The PELs have zero PMA and a $M_{\mathrm{s}}$ of $600 \mathrm{emu} / \mathrm{cm}^{3}$. The exchange constant $\left(\mathrm{A}_{\mathrm{ex}}\right)$ within homogeneous materials is $1.3 \times 10^{6} \mathrm{erg} / \mathrm{cm}$. The interlayer exchange constant $\left(\mathrm{A}_{\mathrm{ex}, \mathrm{IL}}\right)$ between PEL's and $\mathrm{PL}$ or FL is assumed to be $40 \%$ of $A_{\text {ex }}$. Temperature was 
assumed to be zero.

When the electric current is applied to the MTJ, two types of spin torque operate on the magnetizations. First is the Slonczewski spin torque $\left(\mathbf{S T}_{\mathbf{1}}\right)$ working on the magnetizations in the PELs adjacent to the $\mathrm{MgO}$ layer. Spin torque $\boldsymbol{S} \boldsymbol{T}_{\mathbf{1}}$ is the actual driving force for the CIMS. The other one operates on the magnetizations that are not uniform along the thickness direction $\left(\mathbf{S T}_{\mathbf{2}}\right)$, which generally induces the domain wall motion [9-11]. The LandauLifshitz-Gilbert equation was solved, taking into account the two types of spin torque for the micromagnetic modeling,

$$
\begin{aligned}
& \frac{d \mathbf{m}}{d t}=-\gamma_{0} \mathbf{m} \times \mathbf{H}_{e f f}+\alpha \mathbf{m} \times \frac{d \mathbf{m}}{d t}+\mathbf{S T}_{1}+\mathbf{S T}_{2}, \\
& \mathbf{S T}_{1}=-\gamma_{0} \frac{\hbar}{2 e} \frac{P_{1}}{M_{s} d} J_{e} \mathbf{m} \times(\mathbf{m} \times \mathbf{p}), \\
& \mathbf{S T}_{2}=-\frac{P_{2} \mu_{B}}{e M_{s}} J_{e} \mathbf{m} \times\left(\mathbf{m} \times \frac{\partial \mathbf{m}}{\partial z}\right),
\end{aligned}
$$

where $\mathbf{m}$ is the unit vector of magnetization, $\gamma_{0}$ the gyromagnetic ratio, $\alpha$ the Gilbert damping constant $(=0.02)$, $M_{s}$ the saturation magnetization, $d$ the thickness of the layer that experiences $\boldsymbol{S} \boldsymbol{T}_{1}, \boldsymbol{\mu}_{B}$ the Bohr magneton, and $J_{e}$ the current density. The spin-polarizations $P_{1}$ and $P_{2}$ are assumed to be 0.6 and 0.7 , respectively. The unit vector of spin-polarization $\mathbf{p}$ in Eq. (2) is the direction of magnetization of the PEL on the other side of $\mathrm{MgO}$ layer. We assumed effective field terms are zero in $\boldsymbol{S} \boldsymbol{T}_{1}$ and $\boldsymbol{S} \boldsymbol{T}_{2}$ were assumed to be zero.

\section{Results and Discussion}

The switching time of FL $\left(t_{s w}\right)$ was estimated as a function of $J_{e}$ to analyze the effects of magnetic properties on the switching of the CmFL. The change in $t_{s w}$ by variation of magnetic properties will be discussed in terms of the driving force for CIMS, $\boldsymbol{S} \boldsymbol{T}_{1}$, and other magnetic conditions. The value $t_{s w}$ is defined as the time elapsed from the moment when the current is applied, until the perpendicular component of FL magnetization changes its sign. Prior to investigating the change in $t_{s w}$ due to the variation of the properties, the change in $t_{s w}$ due to the insertion of the soft magnetic PELs was first examined. The value of $t_{s w} \mathrm{~s}$ for the MTJ with the default properties were obtained and compared to $t_{s w}$ for the MTJ without PELs, consisting of PL $(20 \mathrm{~nm})|\mathrm{MgO}(1 \mathrm{~nm})| \mathrm{FL}(6 \mathrm{~nm})$, where all other conditions were the same as the default properties. The $t_{s w}$ for a given $J_{e}$ was generally smaller for the switching from an antiparallel (AP) state to parallel (P), Fig. 1(a), than for switching from $\mathrm{P}$ to AP, Fig. 1(b), because the

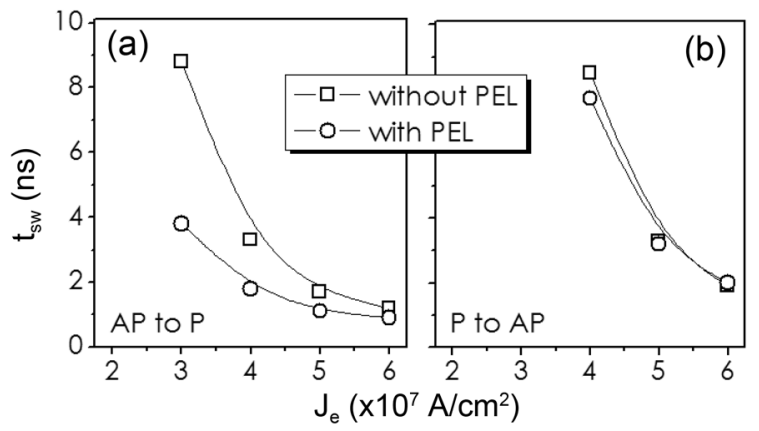

Fig. 1. $t_{s w}$ as a function of $J_{e}$ for switching in MTJs with PELs and without PELs, (a) for the switching of CmFL from AP to $\mathrm{P}$, and (b) for the switching from $\mathrm{P}$ to AP. The lines are guides to the eye.

magnetostatic field generated from the CmPL (PL) assists the switching of CmFL (FL) in the switching from AP to $\mathrm{P}$, while it disturbs in the switching from $\mathrm{P}$ to AP.

The $t_{s w}$ for the switching from AP to $\mathrm{P}$ is significantly reduced by the insertion of PELs, Fig. 1(a), while the $t_{s w}$ for switching from $\mathrm{P}$ to AP remains mostly the same, Fig. 1(b). However, it should be noted that the magnitude of $\boldsymbol{S} \boldsymbol{T}_{1}$ is smaller in the case with PEL's, because the $M_{s}$ of the magnetizations adjacent to the $\mathrm{MgO}$ layer is larger, and the magnitude of $\boldsymbol{S} \boldsymbol{T}_{1}$ is inversely proportional to $M_{s}$, as described in Eq. (2). The result that $t_{s w}$ is reduced or at least retained in spite of the reduction of $\boldsymbol{S} \boldsymbol{T}_{1}$ indicates that the existence of PEL essentially assists the CIMS. Because the PEL's do not have PMA, thus the Top PEL is much more susceptible to excitation. The excitation of the top PEL plays a crucial role in the switching of $\mathrm{CmFL}$, which will be further clarified in the following results on the effect of magnetic property variations. The change in $t_{s w}$ due to the insertion of PELs is less significant in the switching from $\mathrm{P}$ to AP than the opposite switching because the magnetostatic fields on the Top PEL from other layers are very different between the $\mathrm{P}$ state and AP state, and the opposite effect of $\boldsymbol{S} \boldsymbol{T}_{\mathbf{2}}$ according to the current direction [12].

First, $A_{e x, I L}$ was varied from 10 to $80 \%$ of $A_{e x}$. In that range of $A_{e x, I L}$, the PEL magnetizations were exchangecoupled with PMA layers strong enough to be aligned in perpendicular-to-the-plane direction at the remanent state. The result is demonstrated in terms of $t_{s w}$, as a function of $J_{e}$ for various $A_{e x, I L}$ values in Fig. 2. For both switching directions, $t_{s w}$ increased as $A_{e x, I L}$ increased. The stronger the Top PEL was exchange-coupled with the FL, the harder the magnetizations are bound in perpendicular direction. Thus, it became difficult for the Top PEL to be excited out of the perpendicular direction. The large $A_{e x, I L}$ disturbed the switching and subsequently the $t_{s w}$ became 


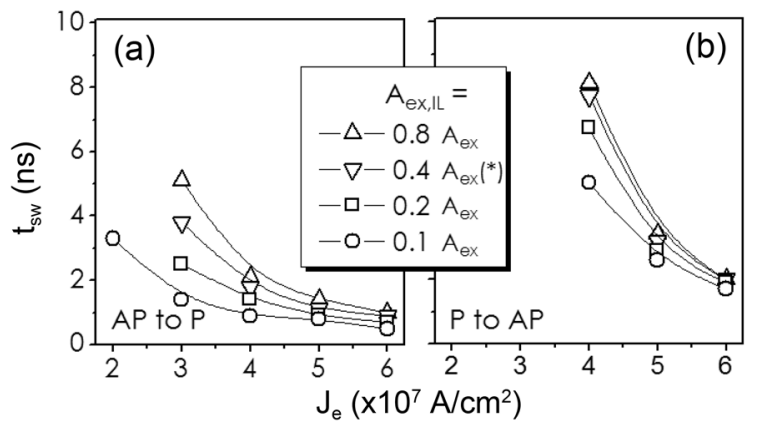

Fig. 2. $t_{s w}$ as a function of $J_{e}$ for various interlayer exchange coupling constants $\left(A_{e x, I L}\right)$, (a) for the switching of CmFL from $\mathrm{AP}$ to $\mathrm{P}$, and (b) for the switching from $\mathrm{P}$ to AP. The lines are guide to the eye. *Results from default set of parameters.

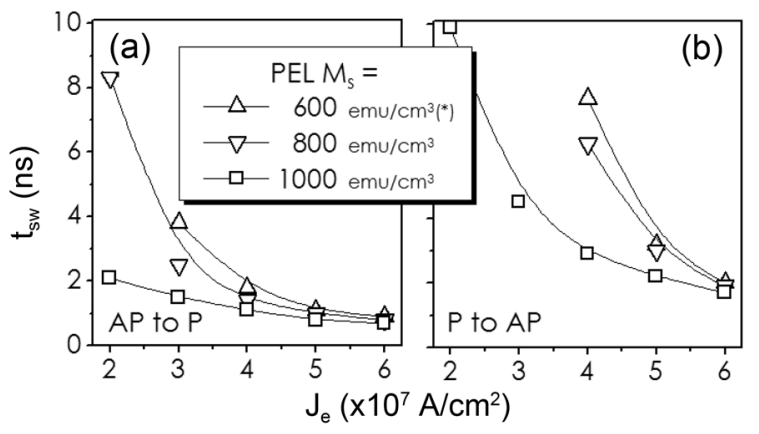

Fig. 3. $t_{s w}$ as a function of $J_{e}$ for various $M_{s}$ of PELs, (a) for the switching of CmFL from AP to P, and (b) for switching from $\mathrm{P}$ to AP. The lines are guides to the eye. *Result from default set of parameters.

longer.

Next, $t_{s w}$ as a function of $J_{e}$ was estimated with changing $M_{s}$ of the PELs as shown in Fig. 3. The value of $t_{s w}$ decreased for both switching directions as $M_{s}$ increased. There are several factors that depend on $M_{s}$ that assisted or disturbed the CIMS, namely a decrease or increase in $t_{s w}$. First, the increase in $M_{s}$ reduced the magnitude of $\boldsymbol{S} \boldsymbol{T}_{1}$, the driving force of the CIMS, as it is inversely proportional to $M_{s}$, as mentioned above. It must disturb the CIMS so that it results in the increase in $t_{s w}$. Another factor depending on $M_{s}$ was the interlayer exchange field, which is also inversely proportional to $M_{s}$. As observed from the results in Fig. 2, a decrease of interlayer exchange coupling assists the CIMS, thus bringing about a decrease in $t_{s w}$. However, the interlayer exchange field is decreased by less than a factor of two in the results in Fig. 3. Comparing with the variation of $t_{s w}$ for different $A_{e x, L L}$ 's in Fig. 2, it is apparent that the decrease in the interlayer exchange field cannot solely induce such significant decreases in $t_{s w}$ in Fig. 3. The last factor is the out-of-plane demagnetizing field of the Top PEL that assists the CIMS in the perpendicular direction as it always acts in the

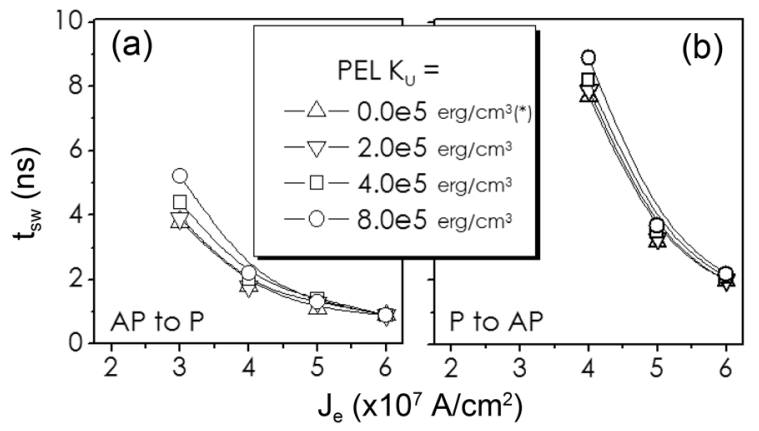

Fig. 4. $t_{s w}$ as a function of $J_{e}$ for various PMAs $\left(K_{u}\right)$ of PELs, (a) for switching of CmFL from AP to P, and (b) for switching from $\mathrm{P}$ to AP. The lines are guides to the eye. *Results from default set of parameters.

opposite direction to the magnetization. Since its magnitude is proportional to $M_{s}$, the magnetizations with larger $M_{s}$ are more easily switched. The decrease in $t_{s w}$ by the increase of $M_{s}$, regardless of the switching direction in Fig. 3 is attributed to all of those effective factors. The reduction in $\boldsymbol{S} \boldsymbol{T}_{\mathbf{1}}$ is less effective to the CIMS, compared with the decrease in $A_{e x, I L}$ and the increase in the demagnetizing field.

Next, the value of $t_{s w}$ was estimated, assuming that the PELs have small PMA. Fe alloys used for the PEL are generally magnetically soft. However, magnetic anisotropy from the interface can be sizable for such a thin layer considered in this work. PMA was thus introduced into the PELs up to $8 \times 10^{5} \mathrm{erg} / \mathrm{cm}^{3}$, which was $40 \%$ of the PMA in PL and FL, and is equivalent to an anisotropy field of approximately 2667 Oe. The $t_{s w}$ increased through the increase in PMA for both switching directions as shown in Fig. 4. However, it did not show significant differences when the PMA was on the order of $10^{5} \mathrm{erg} /$ $\mathrm{cm}^{3}$, the range considered in this work. Moreover, it did not show much variation from the case of zero-PMA, indicating that the magnetic anisotropy in PELs induced at the interface can be negligible even when the anisotropy field is a few thousand Oersted.

\section{Summary}

We performed micromagnetic investigation on the effects of the magnetic properties on CIMS with PMA and soft magnetic PELs. The decrease in interlayer exchange coupling between FL and PEL reduced $t_{s w}$. The increase in $M_{s}$ of PEL assisted the switching of FL, though the magnitude of $\boldsymbol{S} \boldsymbol{T}_{1}$ was reduced. The induced PMA in the PEL did not make significant variations in $t_{s w}$ when it was on the order of $10^{5} \mathrm{erg} / \mathrm{cm}^{3}$. Such results should be taken into account when designing MTJ for MRAM. 


\section{Acknowledgment}

WK and TDL would like to acknowledge the support from the Tera-level Nano-devices (TND) Frontier Project funded by KISTEP. KJL would like to acknowledge the support from the KOSEF through the NRL program funded by the Korean Ministry of Education, Science and Technology (Project No. M10600000198-06J0000-19810).

\section{References}

[1] M. Nakayama, T. Kai, N. Shimomura, M. Amano, E. Kitagawa, T. Nagase, M. Yoshikawa, T. Kishi, S. Ikegawa, and H. Yoda, J. Appl. Phys. 103, 07A710 (2008).

[2] W. H. Butler, X.-G. Zhang, T. C. Schulthess, and J. M. MacLaren, Phys. Rev. B 63, 054416 (2001).
[3] Y. M. Lee, J. Hayakawa, S. Ikeda, F. Matsukura, and H. Ohno, Appl. Phys. Lett. 90, 212507 (2007).

[4] H. Ohomri, T. Hatori, and S. Nakagawa, J. Appl. Phys. 103, 07A991 (2008).

[5] J. C. Slonczewski, J. Magn. Magn. Mater. 159, L1 (1996).

[6] L. Berger, Phys. Rev. B. 54, 9353 (1996).

[7] J. Z. Sun, J. Magn. Magn. Mater. 202, 157 (1999).

[8] Y. Takeda, T. Umezawa, K. Chiba, H. Shoji, and M. Takahashi, J. Magn. Magn. Mater. 152, 243 (1996).

[9] S. Zhang and Z. Li, Phys. Rev. Lett. 93, 127204 (2004).

[10] A. Thiaville, Y. Nakatani, J. Miltat, and Y. Suzuki, Europhys. Lett. 69, 990 (2005).

[11] S. W. Jung and H. W. Lee, J. Magnetics 12, 1 (2007).

[12] W. Kim, T. D. Lee, and K.-J. Lee, Appl. Phys. Lett. 93, 232506 (2008). 